\title{
Gauss Pseudospectral Method for Solving Infinite-Horizon Optimal Control Problems
}

\author{
Divya Garg* \\ William W. Hager ${ }^{\dagger}$ \\ Anil V. Rao \\ University of Florida \\ Gainesville, FL 32611
}

\begin{abstract}
The previously developed Gauss pseudospectral method is extended to the case of nonlinear infinite-horizon optimal control problems. First, the semi-infinite domain $t \in[0,+\infty)$ is transformed to the domain $\tau=[-1,+1)$. The first-order optimality conditions of NLP obtained from the pseudospectral discretization are then presented. These optimality conditions are related to the KKT multipliers of the nonlinear programming problem, leading to an approximation for the costate of the continuous optimal control problem. A key result is that the state and costate are obtained on the entire horizon (including the solution at $t=+\infty$ ). Numerical results show that the method of this paper lead to the ability to determine accurate primal and dual solutions for infinite-horizon optimal control problems.
\end{abstract}

\section{Introduction}

Over the last decade, pseudospectral methods have risen to prominence in the numerical solution of optimal control problems. ${ }^{1-16}$ Pseudospectral methods are a class of direct collocation where the optimal control problem is transcribed to a nonlinear programming problem (NLP) by parameterizing the state and control using global polynomials and collocating the differential-algebraic equations using nodes obtained from a Gaussian quadrature. It is noted that some researchers prefer the term orthogonal collocation, ${ }^{17-19}$ but the terms pseudospectral and orthogonal collocation have the same meaning.

The three most commonly used set of collocation points are Legendre-Gauss (LG), LegendreGauss-Radau (LGR), and Legendre-Gauss-Lobatto (LGL) points. These three sets of points are obtained from the roots of a Legendre polynomial and/or linear combinations of a Legendre polynomial and its derivatives. All three sets of points are defined on the domain $[-1,1]$, but differ significantly in that the LG points include neither of the endpoints, the LGR points include one of the endpoints, and the LGL points include both of the endpoints. In addition, the LGR points are asymmetric relative to the origin and are not unique in that they can be defined using either the initial point or the terminal point. In recent years, the two most well documented pseudospectral methods are the Legendre-Gauss-Lobatto pseudospectral method ${ }^{1,3-5,10,11,20,21}$ (LPM), the LegendreGauss pseudospectral method, ${ }^{13-15,22,23}$ and the Legendre-Gauss-Radau pseudospectral method. ${ }^{24}$

${ }^{*}$ Ph.D. Student, Dept. of Mechanical and Aerospace Engineering. E-mail: divyagarg2002@ufl.edu

${ }^{\dagger}$ Professor. E-mail: hager@math.ufl.edu

${ }^{\ddagger}$ Assistant Professor. E-mail: anilvrao@ufl.edu. Corresponding Author. 
In this paper we develop an extension of the Gauss pseudospectral method to the case of infinitehorizon nonlinear optimal control problems. First, a change of variables is performed to map the semi-infinite domain $t \in[0, \infty)$ into the finite domain $\tau \in[-1,1)$. A transformation is then developed to map the Karush-Kuhn-Tucker (KKT) multipliers of the nonlinear programming problem (NLP) to the costate of the continuous optimal control problem. The method of this paper has the property that the state and the costate are obtained on the entire horizon (including the state and the costate solutions at $t=+\infty)$.

We note that the approach developed in this paper is fundamentally different from the infinitehorizon LGR scheme presented in Ref. 12. Specifically, the approach in Ref. 12 avoids the singularity by neither collocating nor approximating the state at the final point. Thus, unlike the method presented in this paper, the state and the costate in Ref. 12 are not obtained on the entire horizon

\section{Notation}

Throughout the paper, we employ the following notation. $\mathbf{A}^{\top}$ denotes the transpose of a matrix $\mathbf{A}$. Given two matrices $\mathbf{A}$ and $\mathbf{B}$ of the same dimensions, $\langle\mathbf{A}, \mathbf{B}\rangle$ is their dot product:

$$
\langle\mathbf{A}, \mathbf{B}\rangle=\operatorname{trace} \mathbf{A}^{\top} \mathbf{B} .
$$

When $\mathbf{A}$ and $\mathbf{B}$ are vectors, this is the usual vector inner product. If $\mathbf{f}: \mathbb{R}^{n} \rightarrow \mathbb{R}^{m}$, then $\nabla \mathbf{f}$ is the $m$ by $n$ matrix whose $i$-th row is $\nabla f_{i}$. In particular, the gradient of a scalar-valued function is a row vector. If $\phi: \mathbb{R}^{m \times n} \rightarrow \mathbb{R}$ and $\mathbf{X}$ is an $m$ by $n$ matrix, then $\nabla \phi$ denotes the $m$ by $n$ matrix whose $(i, j)$ element is

$$
(\nabla \phi(\mathbf{X}))_{i j}=\frac{\partial \phi(\mathbf{X})}{\partial X_{i j}}
$$

\section{Infinite-Horizon Optimal Control Problem}

Consider the infinite-horizon optimal control problem:

$$
\min J=\int_{0}^{\infty} g(\mathbf{x}(t), \mathbf{u}(t)) d t \quad \text { subject to } \quad \dot{\mathbf{x}}(t)=\mathbf{f}(\mathbf{x}(t), \mathbf{u}(t)), \quad \mathbf{x}(0)=\mathbf{x}_{0},
$$

where $g: \mathbb{R}^{n} \times \mathbb{R}^{m} \rightarrow \mathbb{R}, f: \mathbb{R}^{n} \times \mathbb{R}^{m} \rightarrow \mathbb{R}^{n}$, and $\dot{\mathbf{x}}$ denotes the time derivative of $\mathbf{x}$. We make the change of variables $t=(1+\tau) /(1-\tau)$, thus mapping the half-open interval $[-1,1)$ onto $[0, \infty)$. Next, let $T(\tau)=d t / d \tau=2 /(1-\tau)^{2}$. After changing variables from $t$ to $\tau$, the infinite-horizon optimal control problem becomes

$$
\min J=\int_{-1}^{+1} T(\tau) g(\mathbf{x}(\tau), \mathbf{u}(\tau)) d \tau \quad \text { subject to } \quad \dot{\mathbf{x}}(\tau)=T(\tau) \mathbf{f}(\mathbf{x}(\tau), \mathbf{u}(\tau)), \quad \mathbf{x}(-1)=\mathbf{x}_{0} .
$$

Here $\mathbf{x}(\tau)$ and $\mathbf{u}(\tau)$ denote the state and the control as a function of the new variable $\tau$. the first-order optimality conditions for the finite horizon optimal control problem of Eq. (2) are given as

$$
\begin{aligned}
\dot{\boldsymbol{\lambda}}(\tau) & =-T(\tau) \nabla_{x} H(\mathbf{x}(\tau), \mathbf{u}(\tau), \boldsymbol{\lambda}(\tau)), \quad \boldsymbol{\lambda}(1)=\mathbf{0} \\
\mathbf{0} & =\nabla_{u} H(\mathbf{x}(\tau), \mathbf{u}(\tau), \boldsymbol{\lambda}(\tau))
\end{aligned}
$$

where $\boldsymbol{\lambda}(\tau)$ is the costate for the optimal control problem in Eq. (2) and $H(\mathbf{x}, \mathbf{u}, \boldsymbol{\lambda})=g(\mathbf{x}, \mathbf{u})+$ $\boldsymbol{\lambda}^{\top} \mathbf{f}(\mathbf{x}, \mathbf{u})$ is the Hamiltonian for Eq. (2). 


\section{Infinite-Horizon Gauss Pseudospectral Method}

Consider the Legendre-Gauss collocation points $\left(\tau_{1}, \ldots, \tau_{N}\right)$ on the interval $(-1,1)$ and two additional noncollocated points $\tau_{0}=-1$ (the initial time) and $\tau_{N+1}=1$ (the terminal time, corresponding to $t=+\infty)$. The state is approximated by a polynomial of degree at most $N$ as

$$
\mathbf{x}(\tau) \approx \sum_{j=0}^{N} \mathbf{X}_{j} L_{j}(\tau), \quad L_{j}(\tau)=\prod_{\substack{k=0 \\ k \neq j}}^{N} \frac{\tau-\tau_{k}}{\tau_{j}-\tau_{k}}, \quad j=0, \ldots, N
$$

where $\mathbf{X}_{j} \in \mathbb{R}^{n}$ and $L_{j}$ is a basis of $N$-degree Lagrange polynomials. Notice that the basis includes the function $L_{0}$ corresponding to the initial time $\tau_{0}=-1$, but not a function corresponding to $\tau_{N+1}=+1$. Differentiating the series of Eq. (5) and evaluating at the collocation point $\tau_{i}$ gives

$$
\dot{\mathbf{x}}\left(\tau_{i}\right) \approx \sum_{j=0}^{N} \mathbf{X}_{j} \dot{L}_{j}\left(\tau_{i}\right)=\sum_{j=0}^{N} D_{i j} \mathbf{X}_{j}=\mathbf{D}_{i} \mathbf{X}, \quad i=1, \ldots, N
$$

where $\mathbf{D}_{i}$ is the $i$-th row of $\mathbf{D}$,

$$
D_{i j}=\dot{L}_{j}\left(\tau_{i}\right), \quad \text { and } \quad \mathbf{X}=\left[\begin{array}{c}
\mathbf{X}_{0} \\
\vdots \\
\mathbf{X}_{N}
\end{array}\right]
$$

The rectangular $N \times(N+1)$ matrix $\mathbf{D}$ formed by the coefficients $D_{i j},(i=1, \ldots, N ; j=0, \ldots, N)$ is the Gauss Pseudospectral differentiation matrix since it transforms the state approximations at $\tau_{0}, \ldots, \tau_{N}$ to the derivative of the state approximation at the collocation points $\tau_{1}, \ldots, \tau_{N}$.

Let $\mathbf{U}$ be an $N \times m$ matrix whose $i$-th row $\mathbf{U}_{i}$ is an approximation to the control $\mathbf{u}\left(\tau_{i}\right), 1 \leq$ $i \leq N$. Our discrete approximation to the system dynamics $\dot{\mathbf{x}}(\tau)=T(\tau) \mathbf{f}(\mathbf{x}(\tau), \mathbf{u}(\tau))$ is obtained by evaluating the system dynamics at each collocation point and replacing $\dot{\mathbf{x}}\left(\tau_{i}\right)$ by its discrete approximation $\mathbf{D}_{i} \mathbf{X}$. Hence, the discrete approximation to the system dynamics is

$$
\mathbf{D}_{i} \mathbf{X}=T_{i} \mathbf{f}\left(\mathbf{X}_{i}, \mathbf{U}_{i}\right), \quad 1 \leq i \leq N
$$

where $T_{i}=T\left(\tau_{i}\right)$. The objective function in Eq. (2) is approximated by a Legendre-Gauss quadrature as

$$
J=\int_{-1}^{+1} T(\tau) g(\mathbf{x}(\tau), \mathbf{u}(\tau)) d \tau \approx \sum_{i=1}^{N} w_{i} T_{i} g\left(\mathbf{X}_{i}, \mathbf{U}_{i}\right),
$$

where $w_{i}$ is the quadrature weight associated with $\tau_{i}$. The continuous-time nonlinear finite-horizon optimal control problem of Eq. (2) is then approximated by the following finite-dimensional NLP:

$$
\min \sum_{i=1}^{N} w_{i} T_{i} g\left(\mathbf{X}_{i}, \mathbf{U}_{i}\right) \quad \text { subject to } \quad \mathbf{D}_{i} \mathbf{X}=T_{i} \mathbf{f}\left(\mathbf{X}_{i}, \mathbf{U}_{i}\right), \quad 1 \leq i \leq N, \quad \mathbf{X}_{0}=\mathbf{x}_{0} .
$$

After solving this NLP, the state at the horizon can be estimated by quadrature as follows:

$$
\mathbf{x}(+1)=\mathbf{x}(-1)+\int_{-1}^{+1} T(\tau) \mathbf{f}(\mathbf{x}(\tau), \mathbf{u}(\tau)) d \tau \approx \mathbf{x}_{0}+\sum_{i=1}^{N} w_{i} T_{i} \mathbf{f}\left(\mathbf{X}_{i}, \mathbf{U}_{i}\right)=\mathbf{X}_{N+1} .
$$


Although the change of variables $t=(1+\tau) /(1-\tau)$ must have a singularity at $\tau=+1$, we never evaluate $T(\tau)=2 /(1-\tau)^{2}$ at the singularity in Eq. (8) or Eq. (9), rather we evaluate $T$ at the quadrature points which are all strictly less than 1.

The first-order optimality conditions for Eq. (8), also called the KKT conditions are given as (see Ref. 25 for details)

$$
\begin{aligned}
\mathbf{D}_{i}^{\top} \boldsymbol{\Lambda} & =T_{i} \nabla_{x}\left(w_{i} g\left(\mathbf{X}_{i}, \mathbf{U}_{i}\right)+\left\langle\boldsymbol{\Lambda}_{i}, \mathbf{f}\left(\mathbf{X}_{i}, \mathbf{U}_{i}\right)\right\rangle\right) \\
\mathbf{0} & =T_{i} \nabla_{u}\left(w_{i} g\left(\mathbf{X}_{i}, \mathbf{U}_{i}\right)+\left\langle\boldsymbol{\Lambda}_{i}, \mathbf{f}\left(\mathbf{X}_{i}, \mathbf{U}_{i}\right)\right\rangle\right)
\end{aligned}
$$

$1 \leq i \leq N$. Here $\mathbf{D}_{i}^{\top}$ is the $i$-th row of $\mathbf{D}^{\top}$. Next, analogous to Ref. 26, the first-order optimality conditions of the NLP can be reformed so that they become a discretization of the first-order optimality conditions for the continuous optimal control problem Eq. (2). Let us define the following expressions:

$$
\boldsymbol{\lambda}=\mathbf{W}^{-1} \boldsymbol{\Lambda} \quad \text { and } \quad \mathbf{D}^{\dagger}=-\mathbf{W}^{-1} \mathbf{D}_{1: N}^{\top} \mathbf{W},
$$

were $\mathbf{W}$ is the diagonal matrix whose $i$-th diagonal element is $w_{i}$. Making these substitutions in Eqs. (10) and (11), we can rewrite the optimality conditions as

$$
\begin{aligned}
\mathbf{D}_{i}^{\dagger} \boldsymbol{\lambda} & =-T_{i} \nabla_{x} H\left(\mathbf{X}_{i}, \mathbf{U}_{i}, \boldsymbol{\lambda}_{i}\right), \\
\mathbf{0} & =\nabla_{u} H\left(\mathbf{X}_{i}, \mathbf{U}_{i}, \boldsymbol{\lambda}_{i}\right) .
\end{aligned}
$$

Hence, this transformation makes the discrete optimality conditions look very similar to the continuous Pontryagin minimum principle of Eqs. (3)-(4). There are two basic differences: The continuous derivative $\dot{\boldsymbol{\lambda}}(\tau)$ is replaced by the discrete analog $\mathbf{D}_{i}^{\dagger} \boldsymbol{\lambda}$ and boundary condition $\boldsymbol{\lambda}(+1)=0$ present in the minimum principle is missing from the discrete optimality conditions. Finally, it is important to understand that, although not present in the formulation, the boundary condition $\boldsymbol{\lambda}(+1)=\mathbf{0}$ is incorporated in Eq. (13). The details of this fact are beyond the scope of this paper; see Ref. 25 for details.

The transformed conditions Eqs. (13)-(14) yield estimates for the costate at the collocation points $\tau_{i}$. We can also estimate the costate at $\tau=-1$ by the expression

$$
\lambda_{0}=-\mathbf{D}_{0}^{\top} \Lambda \text {. }
$$

The rationale for this estimate of the initial costate is based on equation (32) in Ref. 27 from which it follows that

$$
\boldsymbol{\lambda}_{0}=\sum_{j=1}^{N} w_{j} T_{j} \nabla_{x} H\left(\mathbf{X}_{j}, \mathbf{U}_{j}, \boldsymbol{\lambda}_{j}\right)
$$

The continuous costate, on the other hand, satisfies

$$
\boldsymbol{\lambda}(-1)=\boldsymbol{\lambda}(+1)-\int_{-1}^{+1} \dot{\boldsymbol{\lambda}}(\tau) d \tau=\int_{-1}^{+1} T(\tau) \nabla_{x} H(\mathbf{x}(\tau), \mathbf{u}(\tau), \boldsymbol{\lambda}(\tau)) d \tau
$$

Hence, the right side of Eq. (15) represents a quadrature approximation to the right side of Eq. (16). We refer to the LG collocation method developed in this section as the infinite-horizon version of the Gauss pseudospectral method. ${ }^{13-15,27,28}$

\section{Equivalence of Integral and Differential Forms}

It has been shown in Ref. 27 that the Gauss pseudospectral method can be written equivalently in either integral or differential form. The differential form is chosen because the differential form leads to a sparse NLP, whereas the integral form leads to a dense NLP. The details of this equivalence are beyond the scope of this paper; see Refs. 27 and 25 for further details. 


\section{Infinite-Horizon Radau Pseudospectral Method}

It is useful to note that, in a manner similar to that given in Section (IV), the continuous infinite-horizon optimal control problem can be approximated using the Legendre-Gauss-Radau (LGR) points. A key difference between LGR and LG points is that the final point (that is, the approximation at $t=+\infty)$ is a variable in the NLP. The details of the derivation of the infinitehorizon LGR pseudospectral method are beyond the scope of this paper; see Ref. 25 for details.

\section{Example}

In this section we consider an example using the aforementioned infinite-horizon Gauss pseudospectral method. The example was solved using the software OptimalPrime ${ }^{29}$ using the NLP solver SNOPT with default optimality and feasibility tolerances.

Consider the following optimal control problem taken from Ref. 12. Denoting $\mathbf{x}(t)=\left[x_{1}(t) x_{2}(t)\right]^{T} \in$ $\mathbb{R}^{2}$ as the state and $u(t) \in \mathbb{R}$ as the control, minimize the cost functional

$$
J=\frac{1}{2} \int_{0}^{\infty}\left(\mathbf{x}^{T} \mathbf{Q} \mathbf{x}+\mathbf{u}^{T} \mathbf{R u}\right) d t
$$

subject to the dynamic constraint

$$
\dot{\mathbf{x}}=\mathbf{A x}+\mathbf{B u}
$$

and the initial condition

$$
\mathbf{x}(0)=\left[\begin{array}{c}
-4 \\
4
\end{array}\right]
$$

The matrices $\mathbf{A}, \mathbf{B}, \mathbf{Q}$, and $\mathbf{R}$ for this problem are given as

$$
\mathbf{A}=\left[\begin{array}{rr}
0 & 1 \\
2 & -1
\end{array}\right], \mathbf{B}=\left[\begin{array}{l}
0 \\
1
\end{array}\right], \quad \mathbf{Q}=\left[\begin{array}{ll}
2 & 0 \\
0 & 1
\end{array}\right], \quad \mathbf{R}=\frac{1}{2}
$$

The exact solution to this problem is

$$
\begin{aligned}
\mathbf{x}(t) & =\exp ([\mathbf{A}-\mathbf{B K}] t) \mathbf{x}(0) \\
u(t) & =-\mathbf{K} \mathbf{x}(t) \\
\boldsymbol{\lambda}(t) & =\mathbf{S x}(t)
\end{aligned}
$$

where $\mathbf{K}$ is the optimal feedback gain and $\mathbf{S}$ is the solution to the algebraic Riccati equation. In this case $\mathbf{K}$ and $\mathbf{S}$ are given, respectively, as

$$
\begin{aligned}
& \mathbf{K}=\left[\begin{array}{ll}
4.828427124746193 & 2.557647291327851
\end{array}\right] \\
& \mathbf{S}=\left[\begin{array}{ll}
6.031273049535752 & 2.414213562373097 \\
2.414213562373097 & 1.278823645663925
\end{array}\right]
\end{aligned}
$$

The optimal control problem of Eqs. (17)-(19) was solved using the infinite-horizon Gauss pseudospectral method (GPM) for $N=3$ to $N=28 \mathrm{LG}$ points by steps of 5 . Suppose now that we define the following maximum absolute errors between the infinite-horizon GPM solution and the 
exact solution:

$$
\begin{aligned}
& e_{x_{1}}=\max _{k} \log _{10}\left|x_{1}\left(\tau_{k}\right)-x_{1}^{*}\left(\tau_{k}\right)\right| \\
& e_{x_{2}}=\max _{k} \log _{10}\left|x_{2}\left(\tau_{k}\right)-x_{2}^{*}\left(\tau_{k}\right)\right| \\
& e_{u}=\max _{k} \log _{10}\left|u\left(\tau_{k}\right)-u^{*}\left(\tau_{k}\right)\right| \\
& e_{\lambda_{1}}=\max _{k} \log _{10}\left|\lambda_{1}\left(\tau_{k}\right)-\lambda_{1}^{*}\left(\tau_{k}\right)\right| \\
& e_{\boldsymbol{\lambda}}=\max _{k} \log _{10}\left|\lambda_{2}\left(\tau_{k}\right)-\lambda_{2}^{*}\left(\tau_{k}\right)\right|
\end{aligned}
$$

The errors are shown in Figs. 1-3. It is seen that all errors decrease linearly until approximately $N=40$, demonstrating a spectral convergence rate.

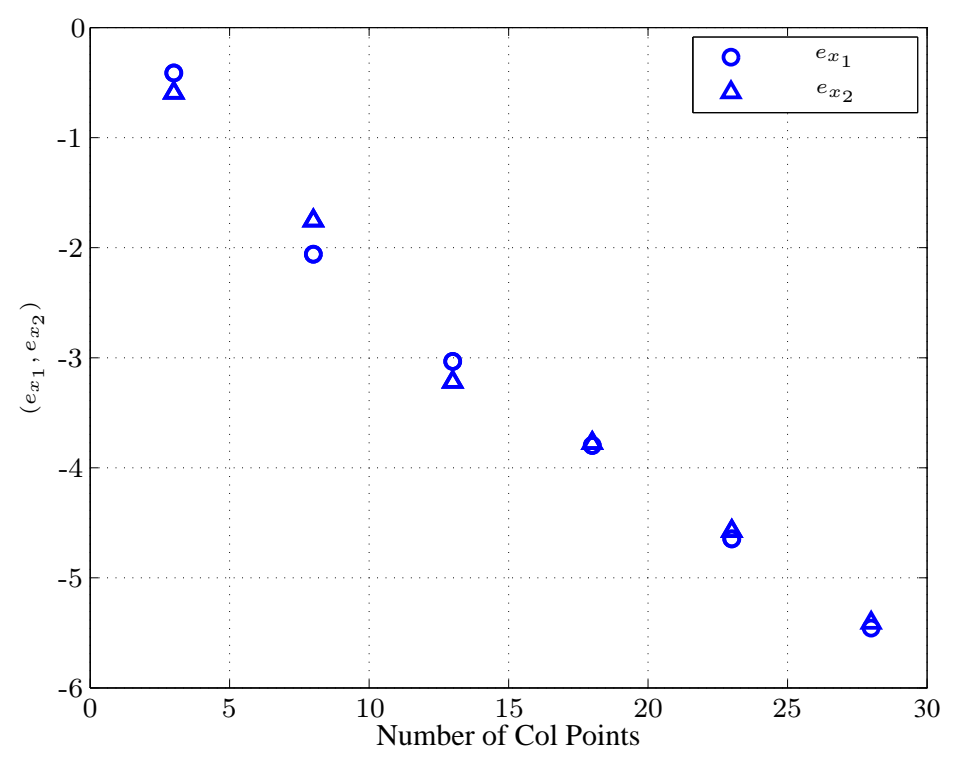

Figure 1: Error in Infinite-Horizon Gauss Pseudospectral State Solution as a function of $N$.

\section{Comparison with Method in Ref. 12}

Ref. 12 develops an approach for solving infinite-horizon optimal control problems using collocation at Legendre-Gauss-Radau points. In particular, the change of variables of variables from $t$ to $\tau$ is used to map the infinite time domain $t \in[0, \infty)$ to the half-open interval $\tau \in[-1,1)$. Because this transformation is singular at $\tau=+1$, it is not possible to collocate the dynamics at $\tau=+1$. To handle this singularity, Fahroo and Ross propose to collocate and discretize at the Legendre-Gauss-Radau (LGR) quadrature points for which the final collocation point is strictly less than 1 (i.e., $\tau_{N}<1$ ). The fundamental difference between the pseudospectral scheme of Ref. 12 and the scheme introduced in this paper is that in Ref. 12, the state is approximated by polynomials of degree $N-1$, while in this paper the state is approximated using polynomials of degree $N$. This change in the degree of the polynomials leads to fundamental differences between the two schemes. For example, since the Lagrange polynomials are of different degrees, the differentiation matrices are completely different. The differentiation matrix used in Ref. 12 is singular, while the matrix $\mathbf{D}_{1: N}$ of Eq. (10) used in this paper is nonsingular. If the control and the initial state $\mathbf{x}_{0}$ are given, then the 


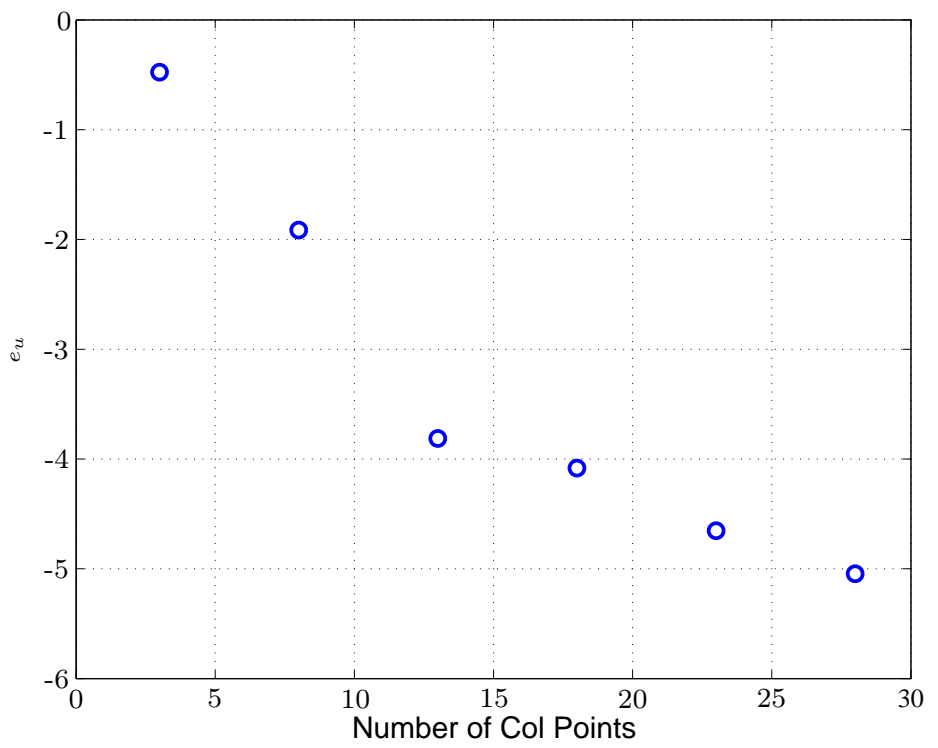

Figure 2: Error in Infinite-Horizon Gauss Pseudospectral Control Solution as a function of $N$.

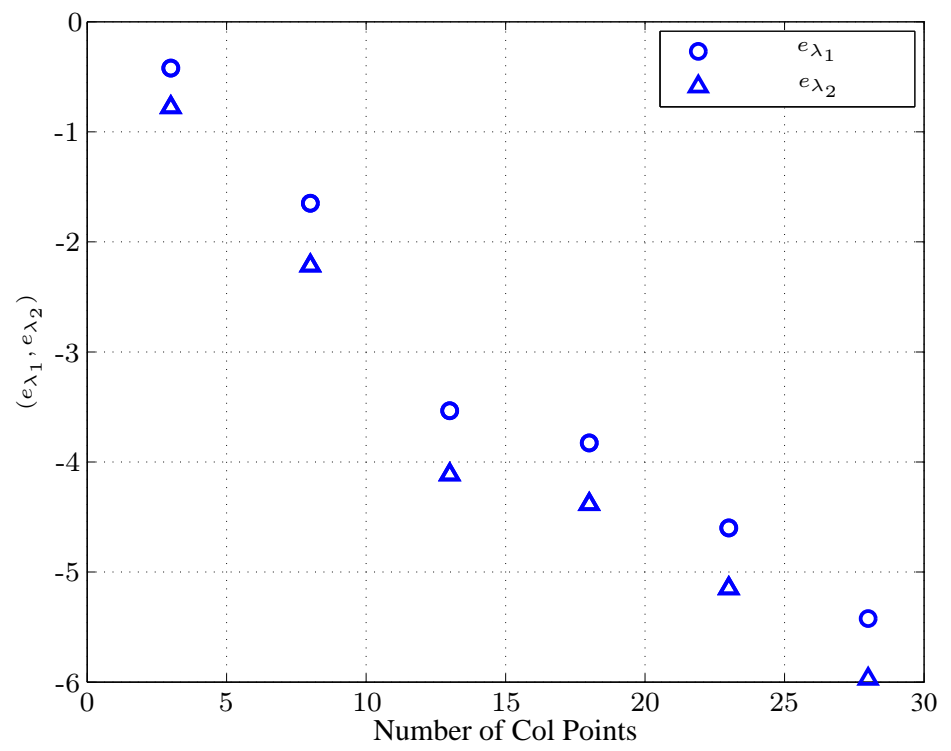

Figure 3: Error in Infinite-Horizon Gauss Pseudospectral Costate Solution as a function of $N$. 
collocated dynamics in Ref. 12 constitutes $N$ equations in $N-1$ unknowns $\mathbf{X}_{2: N}$, an overdetermined system. In contrast, our method constitutes a system of $N$ equations in $N$ unknowns $\mathbf{X}^{\mathrm{LG}}$ where the coefficient matrix $\mathbf{D}_{1: N}$ is invertible. In the approach of Ref. $12, \mathbf{X}_{N+1}$, the estimate of the state at $\tau=+1$, is removed from the problem by using polynomials of degree $N-1$ instead of polynomials of degre $N$. In the approach presented here, however, the state is approximated at $\tau_{i}$, $0 \leq i \leq N+1$. Hence, $\mathbf{X}_{N+1}$, the estimate of the state at the horizon, is part of the pseudospectral scheme. In addition to the fact that the state is estimated at $\tau=+1 \Leftrightarrow t=+\infty$, the ability to estimate the state at $\tau=+1$ is useful in finite-horizon problems when the objective function depends on the state at the terminal time or when there is an endpoint constraint.

\section{Conclusions}

A method has been presented for direct trajectory optimization and costate estimation for an infinite horizon optimal control problem using global collocation at Legendre-Gauss points. A theoretical foundation for the method has been provided. The method allows for solving an infinite horizon optimal control problem and constructing a mapping between the continuous and discrete variables. The results of this paper indicate that the Gauss pseudospectral method described in this paper leads to the ability to determine accurate primal and dual solutions to infinite horizon optimal control problems, including the state and costate solutions at $t=+\infty$.

\section{Acknowledgments}

The authors gratefully acknowledge support for this research from the U.S. Army Research Office under Grant 55173-CI, the U.S. Air Force Research Laboratory under Contract FA8651-08-D-0108, and from the National Science Foundation under Grant 0620286.

\section{References}

\footnotetext{
${ }^{1}$ Elnagar, G., Kazemi, M., and Razzaghi, M., "The Pseudospectral Legendre Method for Discretizing Optimal Control Problems," IEEE Transactions on Automatic Control, Vol. 40, No. 10, 1995, pp. 1793-1796.

${ }^{2}$ Elnagar, G. N. and Razzaghi, M., "A Collocation-Type Method for Linear Quadratic Optimal Control Problems," Optimal Control Applications and Methods, Vol. 18, No. 3, 1997, pp. 227-235.

${ }^{3}$ Fahroo, F. and Ross, I. M., "A Spectral Patching Method for Direct Trajectory Optimization," Journal of Astronautical Sciences, Vol. 48, No. 2-3, Apr.-Sept. 2000, pp. 269-286.

${ }^{4}$ Fahroo, F. and Ross, I. M., "Costate Estimation by a Legendre Pseudospectral Method," Journal of Guidance, Control, and Dynamics, Vol. 24, No. 2, 2001, pp. 270-277.

${ }^{5}$ Ross, I. M. and Fahroo, F., Lecture Notes in Control and Information Sciences, chap. Legendre Pseudospectral Approximations of Optimal Control Problems, Springer-Verlag, 2003.

${ }^{6}$ Rao, A. V., "Extension of a Pseudospectral Legendre Method for Solving Non-Sequential Multiple-Phase Optimal Control Problems," AIAA Guidance, Navigation, and Control Conference, AIAA Paper 2003-5634, Austin, Texas, August 11-14 2003.

${ }^{7}$ Williams, P., "Jacobi Pseudospectral Method for Solving Optimal Control Problems," Journal of Guidance, Control, and Dynamics, Vol. 27, No. 2, 2004, pp. 293-297.

${ }^{8}$ Williams, P., "Application of Pseudospectral methods for Receding Horizon Control," Journal of Guidance, Control, and Dynamics, Vol. 27, No. 2, 2004, pp. 310-314.

${ }^{9}$ Williams, P., "Hermite-Legendre-Gauss-Lobatto Direct Transcription Methods in Trajectory Optimization," Journal of Guidance, Control, and Dynamics, Vol. 32, No. 4, 2009, pp. 1392-1395.

${ }^{10}$ Ross, I. M. and Fahroo, F., "Pseudospectral Knotting Methods for Solving Optimal Control Problems," Journal of Guidance, Control, and Dynamics, Vol. 27, No. 3, 2004, pp. 397-405.

${ }^{11}$ Fahroo, F. and Ross, I. M., "On Discrete-Time Optimality Conditions for Pseudospectral Methods," $A I A A$ Guidance, Navigation, and Control Conference, AIAA Paper 2006-6304, Keystone, Colorado, August 2006.
} 
${ }^{12}$ Fahroo, F. and Ross, I. M., "Pseudospectral Methods for Infinite-Horizon Nonlinear Optimal Control Problems," Journal of Guidance, Control, and Dynamics, Vol. 31, No. 4, 2008, pp. 927-936.

${ }^{13}$ Benson, D. A., A Gauss Pseudospectral Transcription for Optimal Control, Ph.D. thesis, MIT, 2004.

${ }^{14}$ Benson, D. A., Huntington, G. T., Thorvaldsen, T. P., and Rao, A. V., "Direct Trajectory Optimization and Costate Estimation via an Orthogonal Collocation Method," Journal of Guidance, Control, and Dynamics, Vol. 29, No. 6, November-December 2006, pp. 1435-1440.

${ }^{15}$ Huntington, G. T., Advancement and Analysis of a Gauss Pseudospectral Transcription for Optimal Control, Ph.D. thesis, Department of Aeronautics and Astronautics, Massachusetts Institute of Technology, 2007.

${ }^{16}$ Kameswaran, S. and Biegler, L. T., "Convergence Rates for Direct Transcription of Optimal Control Problems Using Collocation at Radau Points," Computational Optimization and Applications, Vol. 41, No. 1, 2008 , pp. 81-126.

${ }^{17}$ Reddien, G. W., "Collocation at Gauss Points as a Discretization in Optimal Control," SIAM Journal on Control and Optimization, Vol. 17, No. 2, March 1979.

${ }^{18}$ Cuthrell, J. E. and Biegler, L. T., "On the Optimization of Differential-Algebraic Processes," AIChe Journal, Vol. 33, No. 8, August 1987, pp. 1257-1270.

${ }^{19}$ Cuthrell, J. E. and Biegler, L. T., "Simultaneous Optimization and Solution Methods for Batch Reactor Control Profiles," Computers and Chemical Engineering, Vol. 13, No. 1/2, 1989, pp. 49-62.

${ }^{20}$ Fahroo, F. and Ross, I. M., "Advances in Pseudospectral Methods," AIAA Guidance, Navigation, and Control Conference, AIAA Paper 2008-7309, Honolulu, Hawaii, August 2008.

${ }^{21}$ Ross, I. M. and Fahroo, F., "Convergence of the Costates Does Not Imply Convergence of the Controls," Journal of Guidance, Control, and Dynamics, Vol. 31, No. 5, 2008, pp. 1492-1496.

${ }^{22}$ Huntington, G. T., Benson, D. A., and Rao, A. V., "Optimal Configuration of Tetrahedral Spacecraft Formations," The Journal of the Astronautical Sciences, Vol. 55, No. 2, April-June 2007, pp. 141-169.

${ }^{23}$ Huntington, G. T. and Rao, A. V., "Optimal Reconfiguration of Spacecraft Formations Using the Gauss Pseudospectral Method," Journal of Guidance, Control, and Dynamics, Vol. 31, No. 3, May-June 2008 , pp. $689-698$.

${ }^{24}$ Garg, D., Patterson, M. A., Darby, C. L., Francolin, C., Huntington, G. T., Hager, W. W., and Rao, A. V., "Direct Trajectory Optimization and Costate Estimation of Finite-Horizon and Infinite-Horizon Optimal Control Problems Using a Radau Pseudospectral Method," Computational Optimization and Applications, 2009, DOI: 10.1007/s10589-009-9291-0.

${ }^{25}$ Garg, D., Hager, W. W., and Rao, A. V., "Pseudospectral Methods for Infinite-Horizon Optimal Control Problems," Automatica, submitted, June 2010. Currently available at http://vdol.mae.ufl.edu/.

${ }^{26}$ Hager, W. W., "Runge-Kutta methods in optimal control and the transformed adjoint system," Numer. Math., Vol. 87, 2000, pp. 247-282.

${ }^{27}$ Garg, D., Patterson, M. A., Hager, W. W., Rao, A. V., Benson, D. A., and Huntington, G. T., "A Unified Framework for the Numerical Solution of Optimal Control Problems Using Pseudospectral Methods," Automatica, December 2009, conditionally accepted.

${ }^{28}$ Rao, A. V., Benson, D. A., Darby, C., Patterson, M. A., Francolin, C., Sanders, I., and Huntington, G. T., "Algorithm 902: GPOPS, A MATLAB Software for Solving Multiple-Phase Optimal Control Problems Using the Gauss Pseudospectral Method," ACM Transactions on Mathematical Software, Vol. 37, No. 2, 2010, pp. 22:1-22:39.

${ }^{29}$ Patterson, M. A., "OptimalPrime: An Open-Source MATLAB Software for Solving Non-Sequential MultiplePhase Optimal Control Problems Using Pseudospectral Methods," Dept. of Mechanical and Aerospace Engineering, University of Florida, August 2008. Available via Download at http://code.google.com/p/optimalprime. 